\title{
Deposition Order Controls the First Stages of a Metal-Organic Coordination Network on an Insulator Surface
}

\author{
Lukas Schüller, ${ }^{\dagger}$ Ville Haapasilta, ${ }^{\ddagger}$ Stefan Kuhn, ${ }^{\dagger}$ Hugo Pinto, $^{\dagger}$ Ralf Bechstein, ${ }^{\dagger}$ Adam S. Foster, ${ }^{*}, \S$ \\ and Angelika Kühnle*, \\ ${ }^{\dagger}$ Institute of Physical Chemistry, Johannes Gutenberg University Mainz, Duesbergweg 10-14, 55099 Mainz, Germany \\ ${ }^{\ddagger}$ COMP, Department of Applied Physics, Aalto University, Otakaari 1, FI-00076 Helsinki, Finland \\ ${ }^{\S}$ Division of Electrical Engineering and Computer Science, Kanazawa University, Kanazawa 920-1192, Japan
}

\begin{abstract}
We report on first stages toward the formation of a surface-confined metal-organic coordination network (MOCN) by sequential deposition of biphenyl-4,4'-dicarboxylic acid and iron atoms on the surface of a bulk insulator, calcite (10.4). The influence of the deposition order on the structure formation is studied by noncontact atomic force microscopy operated in ultrahigh vacuum at room temperature. It is found that sequential deposition facilitates MOCN formation when the organic linker molecules are first adsorbed on the surface, followed by iron deposition. This observation is explained by first-principles computations, indicating that the
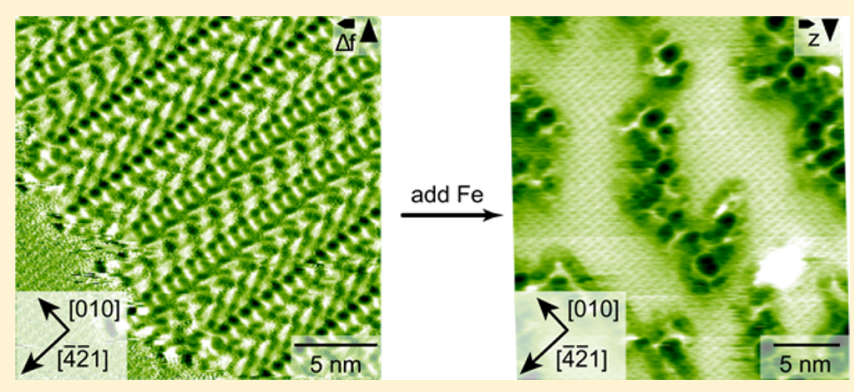
metal-molecule interaction dominates over the molecule-molecule interaction on the surface. The observed MOCN islands are elongated in the [010] substrate direction, demonstrating a templating effect of the underlying substrate. This experimental finding is confirmed by calculations suggesting that the MOCN network matches the calcite lattice periodicity in the [010] direction but not in the [ $\overline{42} 1]$ direction. This work, thus, demonstrates the decisive influence of both deposition order and lattice matching on the formation of an extended MOCN on a bulk insulator surface.
\end{abstract}

\section{INTRODUCTION}

Metal-organic coordination networks (MOCNs) constitute an interesting class of materials in which organic linkers are interconnected by coordinating metal atoms. ${ }^{1,2}$ These regular arrays of metal centers provide novel magnetic ${ }^{3,4}$ or catalytic properties ${ }^{5}$ that are highly interesting for applications such as gas sensing, data storage, or catalysis. ${ }^{6-9}$ Recently, MOCNs have also been discussed as promising candidates for a new class of topological insulators. ${ }^{10}$ Varying the structure of the constituting organic linkers ${ }^{11}$ allows for tuning the size of the resulting nanocavities in a well-controlled manner, ${ }^{12}$ which can be used for tailoring the network pore size, e.g., for subsequent deposition of guest species. ${ }^{13}$ Because of the inherent flexibility and the wide range of promising applications, MOCNs have attracted considerable attention in the past decade with a focus on elucidating the formation procedures that control the final MOCN structure. For example, the influence of thermodynamic versus kinetic control on the structural dimensionality has been addressed with specially designed molecular building blocks on $\mathrm{Au}(111)$ at various temperatures. ${ }^{14}$ The metal versus ligand concentration ratio is known to be decisive for the resulting structure in some systems, e.g., terephthalic acid ${ }^{15,16}$ as well as trimellitic acid ${ }^{17}$ coadsorbed to $\mathrm{Fe}$ on $\mathrm{Cu}(100)$. This is in contrast to MOCN formation of biphenyldicarboxylic acid and $\mathrm{Fe}$ on the same surface, where this ratio appears to be insignificant for the resulting structure. ${ }^{16}$ When intrinsically available adatoms of the supporting surface are incorporated in the network, increasing the substrate temperature can be employed to tune the metal-ligand concentration ratio. ${ }^{18,19}$ For porphyrin-based networks, postdeposition annealing has been shown to result in the metalation of the molecular building block, consuming the Co atoms initially responsible for the formation of the coordination bond. ${ }^{20}$ Simultaneous metalation and coordination has been presented for a carbonitrile-functionalized porphyrin derivative on an insulating thin film of boron nitride. ${ }^{21}$

Using an electrically insulating substrate is of interest when aiming for decoupling the metallic centers from the underlying substrate, which is mandatory for sustaining the electronic, magnetic, and catalytic activity of the metal nodes. ${ }^{8,22,23}$ However, no MOCN prepared on a bulk insulator surface has been presented to date. This might be, in part, due to the fact that insulator surfaces are experimentally more challenging, since standard surface science techniques such as scanning tunneling microscopy cannot be applied. Besides this technical issue, insulator surfaces pose a further challenge when aiming for MOCN formation, which originates from the comparatively low surface energy as compared to metal surfaces. Depositing metal atoms on prototypical insulators such as $\operatorname{KBr}(001)^{24,25}$ typically result in dewetting of the metal atoms and Volmer-

Received: May 10, 2016

Revised: June 17, 2016

Published: June 18, 2016 
Weber growth ${ }^{26}$ of large, inert metal clusters. In a recent work, a covalently interlinked MOCN has been prepared on a thin insulating film of $\mathrm{NaCl}$ on $\mathrm{Ag}(001)$, demonstrating the influence of the molecule-metal ratio on the resulting polymeric film. ${ }^{27}$ From these studies, it is expected that MOCN formation on insulating surfaces requires paying special attention to system parameters such as surface energy as well as preparation conditions, e.g., deposition order.

In this work we present the first stages of MOCN formation, for the first time, on a bulk insulator surface, namely calcite (10.4). A model of the (10.4) cleavage plane is shown in Figure 1a. The MOCN is created using biphenyl-4,4'-dicarboxylic acid (a)

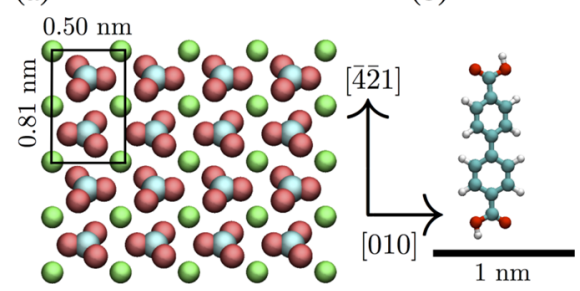

Figure 1. Model of (a) the calcite (10.4) surface and (b) the BPDCA molecule used in this study. The scale bar applies to both subsets. Ca is depicted in green, carbon in blue, oxygen in red, and hydrogen in white.

(BPDCA, see Figure $1 \mathrm{~b}$ ) and iron (Fe). We investigate the influence of the deposition order on the structure formation by noncontact atomic force microscopy (NC-AFM) operated in ultrahigh vacuum (UHV), illustrating that MOCN formation sensitively depends on the preparation condition. This finding is supported by computations at the density functional level of quantum theory, which reveal a hierarchy of interaction strengths, indicating that the metal-molecule interaction is favored over the intermolecular interaction on the surface, thus allowing MOCN formation when the deposition order is favorable. An analysis of the obtained NC-AFM images elucidates the templating effect of the underlying substrate, which is confirmed by corresponding calculations. Thus, this work discloses the decisive influence of the deposition order and precise matching of the molecule/substrate system for creating an extended network on a bulk insulator surface.

\section{METHODS}

Experimental Part. All experiments presented here were performed in a UHV chamber with a base pressure better than $10^{-10}$ mbar. Calcite crystals were obtained from Korth Kristalle (Altenholz, Germany) and cleaved in situ. The BPDCA molecules were purchased and prepared as described elsewhere. $^{28}$ For sublimation, the crucible was heated to a temperature of $177{ }^{\circ} \mathrm{C}$, while the calcite sample was kept at room temperature. A sublimation time of 5 or $10 \mathrm{~min}$ resulted in coverages of roughly a quarter and half a monolayer, respectively. A complete monolayer of BPDCA corresponds to roughly 0.7 molecules $/ \mathrm{nm}^{2}$. Iron was sublimated from a metal evaporator from MBE Komponenten (Weil der Stadt, Germany). For the results shown here, an iron sublimation time of $10 \mathrm{~s}$ and a sublimation temperature of $1150{ }^{\circ} \mathrm{C}$ were chosen. NC-AFM imaging was performed with a UHV VT AFM from Omicron Nanotechnology (Taunusstein, Germany). All images shown in this work were taken with the sample held at room temperature.
Theoretical Part. To obtain further molecular-level insight into the process, we performed density functional theory (DFT) computations. In order to balance accuracy and efficiency, our computational approach was twofold: we used both static geometry optimization calculations at $T=0 \mathrm{~K}$ and first-principles molecular dynamics simulations at room temperature. In all computations, the PBE functional ${ }^{29}$ was used together with Grimme's D3 dispersion correction. ${ }^{30}$ The VASP code ${ }^{31}$ with PAW pseudopotentials ${ }^{32}$ and a plane-wave basis set with an energy cutoff of $450 \mathrm{eV}$ were used to perform optimization calculations. The Brillouin zone was sampled using a Monkhorst-Pack $2 \times 2 \times 1$ grid. $^{33}$ Optimizations of larger systems and first-principles molecular dynamics simulations were performed using the CP2K program suite. ${ }^{34}$ In all $\mathrm{CP} 2 \mathrm{~K}$ computations the dual Gaussian and plane-waves basis set approach was used at the DZVP-MOLOPT/500 Ry level of theory ${ }^{35}$ together with GTH pseudopotentials ${ }^{36}$ for the core electrons. All CP2K computations were $\Gamma$-point calculations. In the molecular dynamics simulations, a time step of $0.5 \mathrm{fs}$ was used. Calcite bond lengths, angles, and surface unit vectors were carefully checked for convergence, and comparisons between the two codes showed essentially equivalent results that also agree with the experimental values within a few percent margin of error.

The calcite (10.4) surface was modeled using a four-layer slab separated by a vacuum region of roughly $1.5 \mathrm{~nm}$ to prevent the interaction between surfaces. During the structure optimization the atoms in the bottom two layers of the calcite slab were fixed while all the other atoms were allowed to move until the forces acting in each atom were less than $0.01 \mathrm{eV} / \AA$. The adsorption energies of single entities were calculated as $E_{\text {ads }}=E_{\text {complex }}-\left(E_{\text {calcite }}+E_{\text {adsorbate }}\right)$ where $E_{i}$ is the absolute value of electronic ground-state energy of the complete system ( $i=$ complex), bare calcite surface $(i=$ calcite $)$, and an isolated adsorbate in vacuum ( $i=$ adsorbate). When there was more than one entity on the surface, we used the interaction energies $E_{\text {int }}=E_{\text {form }}-\sum E_{\text {ads }}$ to estimate the binding between the adsorbates, where the formation energy of the whole complex is defined as $E_{\text {form }}=E_{\text {complex }}-\left(E_{\text {calcite }}+\sum E_{\text {adsorbate }}\right)$. The migration barriers were calculated using the nudged elastic band method (NEB). ${ }^{37}$

\section{RESULTS AND DISCUSSION}

Depositing Only BPDCA. When depositing a submonolayer of BPDCA onto calcite (10.4) held at room temperature, molecular islands are observed on the surface as shown in the NC-AFM images given in Figure 2. These islands are formed by molecules arranged in combination of side-by-side and head-totail fashion as has been described previously. ${ }^{28}$

Calculations reveal that for a single BPDCA molecule the potential energy surface above the calcite (10.4) surface is very flat and contains several local minima, with no clearly preferred adsorption position and orientation suggested from the ground state energies. This is due to the nature of the compound: especially the carbonate group atoms can easily adopt a multitude of configurations with very small barriers. The molecule-surface interaction is based on hydrogen bonding, with the carboxylic group of the molecule $(-\mathrm{COOH})$ keen to donate and calcite, as an alkaline surface, readily accepting the bond via the carbonate group oxygen atoms. According to the calculations, the adsorption energy is within the range of 1.6$2.2 \mathrm{eV}$, which is in good agreement with the observed room temperature adsorption. Several orientations of the molecule on 


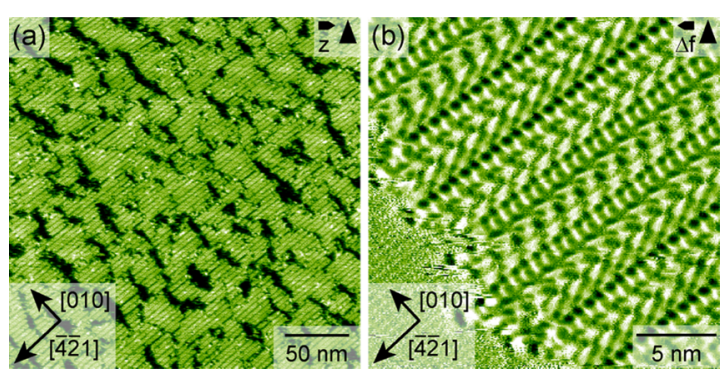

Figure 2. NC-AFM images of molecular islands formed by BPDCA molecules on calcite (10.4) in the absence of iron. The coverage is roughly 0.9 ML. An inner stripe structure oriented along the [421] direction is revealed.

the surface yield very similar adsorption energies: the common denominator in these geometries is the convenient hydrogen bonding between the molecule and the surface as described above. No proton transfer reactions are observed in the geometries where isolated molecules are on the surface (see Figure $3 a-c)$. To assess the effect of temperature on the

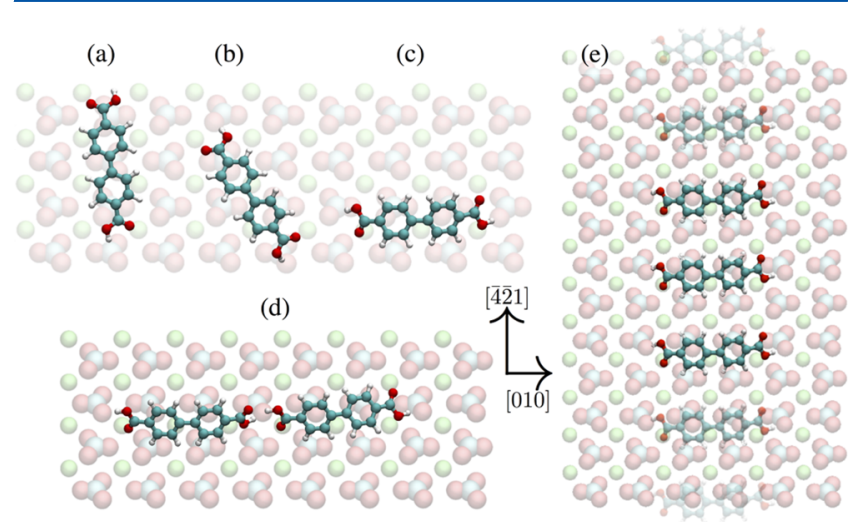

Figure 3. BPDCA molecules on calcite (10.4) in the absence of Fe. ${ }^{28}$ Optimized geometries of isolated molecules in different orientations with respect to the surface: (a) along the [ $\overline{42} 1]$ surface direction, (b) diagonally, and (c) along the [010] surface direction. A hydrogenbonding dimer structure is shown in (d). In (e) a molecular row running in the $[\overline{42} 1]$ direction composed of side-by-side laying molecules along the [010] direction is shown.

system, we performed first-principles molecular dynamics simulations at room temperature with the molecule initially aligned either along [010] or [ $\overline{42} 1]$ direction. In the simulation runs, no clear preference with respect to the alignment was observed and no proton transfers occurred, suggesting that the isolated BPDCA molecules are able to diffuse easily on the surface. This is again in agreement with the experimental observations of molecular self-assembly (Figure 2). ${ }^{28}$

While performing a full first-principles investigation on the observed molecular self-assembly patterns is beyond the scope of this work, it is instructive to consider the molecule-molecule interactions on the surface. Toward this end, we investigated the molecular binding energetics where the carboxyl groups of the neighboring molecules are able to interact directly and where the carboxyl groups can only interact with the surface. When the molecules can hydrogen-bond directly, the molecule-molecule interaction energy is substantial-in this case $2.9 \mathrm{eV}$ (the bonding geometry is shown in Figure 3d). In the other case where the molecules are side-by-side, the molecular interaction is much weaker, roughly $0.8 \mathrm{eV}$ (the bonding geometry is shown in Figure 3e). These values are likely to be upper estimates for the interaction strengths but are nevertheless indicative of the relevant interaction energy range.

Depositing Only Fe. Depositing Fe on a freshly cleaved calcite (10.4) surface results in homogeneously distributed Fe clusters as shown in Figure 4a. After a sublimation time of $10 \mathrm{~s}$
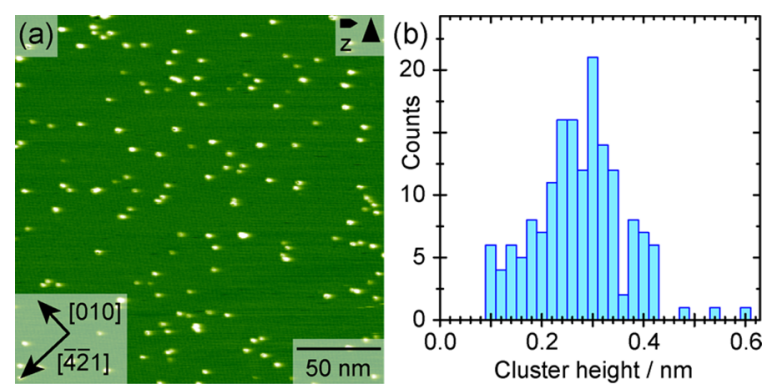

Figure 4. (a) Metal clusters on calcite (10.4) after a sublimation time of $10 \mathrm{~s}$ at a metal evaporator temperature of $1150{ }^{\circ} \mathrm{C}$, resulting in a cluster density of approximately 0.003 clusters $/ \mathrm{nm}^{2}$. (b) The corresponding cluster height histogram.

at a metal evaporator temperature of $1150{ }^{\circ} \mathrm{C}$, a cluster density of approximately 0.003 clusters $/ \mathrm{nm}^{2}$ is revealed. The average apparent height of the clusters is about $0.3 \mathrm{~nm}$ as can be deduced from the height histogram given in Figure $4 \mathrm{~b}$. The observed cluster heights compare well with one to three layers thick Fe clusters assuming an iron atom diameter of $0.25 \mathrm{~nm}$ as in $\alpha$-Fe. The total amount of iron on the surface is difficult to estimate, since the apparent lateral size of the clusters in the NC-AFM image results from a convolution between cluster and NC-AFM tip. A two-layer-thick cluster with a radius of $1 \mathrm{~nm}$ contains about $100 \mathrm{Fe}$ atoms. In this case, we would arrive at approximately $0.3 \mathrm{Fe}$ atoms $/ \mathrm{nm}^{2}$ at the above-mentioned cluster density. The diffusive clustering behavior of iron on calcite (10.4) is also supported by the calculations: an iron monomer adsorbs onto the calcite surface with an adsorption energy of over $1 \mathrm{eV}$ and a migration barrier between $\mathrm{Ca}-\mathrm{Ca}$ bridge sites of $0.6 \mathrm{eV}$. This low barrier implies that iron atoms are able to diffuse on the surface and can form larger clusters at room temperature.

Coadsorption of BPDCA and Fe. For creating a MOCN, we sequentially adsorbed both BPDCA and Fe onto freshly cleaved calcite. First, we investigated the influence of the deposition order. When first depositing the molecules (10 min sublimation time at $177^{\circ} \mathrm{C}$ ) and after that depositing $\mathrm{Fe}$ atoms (10 s sublimation time at $1150{ }^{\circ} \mathrm{C}$ ), we observed a significant morphology change compared to the previous results (see Figure 5a). The observed structure differs substantially from what has been revealed before for the deposition of the individual components (Figures 2 and 4). We attribute this morphology change to the first stages of MOCN formation, composed of $\mathrm{Fe}$ atoms and BPDCA molecules, as will be discussed below in detail. When reversing the deposition order (Fe first, then BPDCA), phase separation is obtained (see Figure $5 b$ ). Here, we can recognize molecular islands with an inner stripe structure oriented along the $[\overline{42} 1]$ direction. These molecular islands closely resemble the structures obtained when depositing the molecules solely. Besides the molecular islands, bright features resembling solely deposited metallic clusters are observed. Thus, we can readily conclude that the deposition order greatly influences the resulting structure. When first depositing the molecules and then the metal atoms, a 


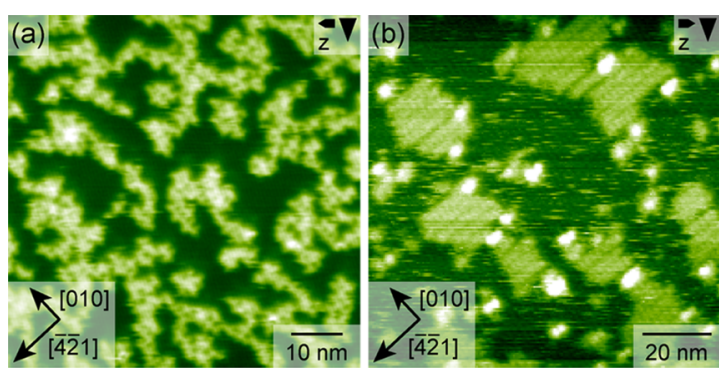

Figure 5. Influence of the deposition order. (a) When first depositing BPDCA molecules and subsequently $\mathrm{Fe}$ atoms, a new arrangement attributed to the first stages of an MOCN is obtained. (b) When reversing the order, large metallic clusters are observed to coexist with molecular islands (compare Figure 2).

qualitatively new structure is obtained. This is in contrast to the reverse deposition order, where the previously obtained structures for the individual components are observed to coexist on the surface.

Simulations demonstrate that on the calcite surface even a single $\mathrm{Fe}$ atom is able to induce a proton transfer from the BPDCA molecule to the metal, both at $0 \mathrm{~K}$ and at room temperature. The same behavior is obtained with an iron cluster of six atoms-an estimate for the typical size of the $\mathrm{Fe}$ clusters in the observed MOCNs. Figure 6 shows the optimized

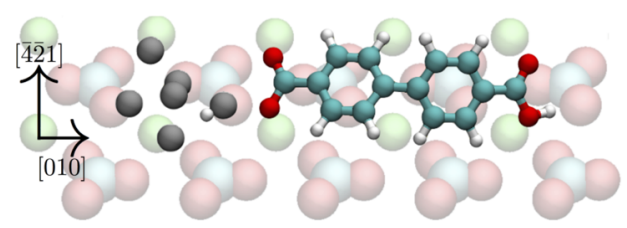

Figure 6. BPDCA molecule on calcite (10.4) with a $\mathrm{Fe}_{6}$ cluster. The molecule is aligned along the [010] surface direction. The metalmolecule interaction is found to be stronger than the moleculemolecule interaction.

geometry of the BPDCA molecule coadsorbed with a $\mathrm{Fe}_{6}$ cluster on the calcite (10.4) surface. The interaction energy between the molecule and the metal cluster ranges from 3.2 to $3.6 \mathrm{eV}$ (depending on the reference adsorption geometry), indicating a rather strong interaction. Importantly, the interaction between the molecule and the metal cluster is stronger than the interaction between two molecules on the surface. Furthermore, according to the calculations the interaction energy of two iron atoms in an iron dimer is on the order of $4 \mathrm{eV}$, and creating larger clusters will only increase the energy gain: the formation energy of $\mathrm{Fe}_{6}$ on the surface is of the order of $28 \mathrm{eV}$. Together with the calculated small migration barriers for the on-surface $\mathrm{Fe}$ atom diffusion, these results predict that when iron is deposited on the surface it will form clusters. Furthermore, the interaction between a BPDCA molecule and a Fe cluster is not strong enough to break the cluster. Thus, this deposition order (first iron, then molecule) will lead to the coexistence of the clusters and the molecular self-assembly pattern. If the deposition order is reversed (first molecules, then iron), the calculated interaction strengths suggest that metal-molecule interaction will prevail over molecule-molecule interaction-with the consequences revealed by our AFM imaging showing the first stages of an MOCN.

To further elucidate the molecular-scale arrangement of the observed structure presumed to be the first stages of an MOCN (Figure 5a), we obtained high-resolution NC-AFM images of the sample where the molecules were deposited before the metal: these are shown in Figure 7. Here, the underlying calcite crystal is resolved with atomic resolution along with an inner structure of the network. Interestingly, the network structure typically extends around $10-20 \mathrm{~nm}$ in [010] direction, while the extent in the $[\overline{42} 1]$ direction is much shorter (see Figure 7a). A closer look at the observed network reveals that the inner structure resembles a rectangular mesh of linker lines with nodes (Figure $7 \mathrm{~b}$ ). The nodes are tentatively assigned to $\mathrm{Fe}$, which are interlinked by BPDCA molecules, as illustrated in Figure $7 \mathrm{c}$. The measured repeat (node-to-node) distance is 2 $\mathrm{nm}$, corresponding to four substrate lattice units along the [010] direction. In the $[\overline{42} 1]$ direction, we rarely observe more than two nodes within a line. In this direction, the node-tonode distance of $2 \mathrm{~nm}$ is not in registry with the underlying substrate unit cell dimension, which is $0.8 \mathrm{~nm}$.

To investigate the possible templating effect of the calcite surface on the resulting MOCN, we tried to create a part of the network in the $[010]$ and $[\overline{42} 1]$ lattice directions in our computations. In the [010] direction this was successfully achieved by forcing another proton transfer reaction (see Figure $8 \mathrm{a}$ ). The second proton transfer reaction lowers the ground state energy of the system by roughly $0.8 \mathrm{eV}$, yielding
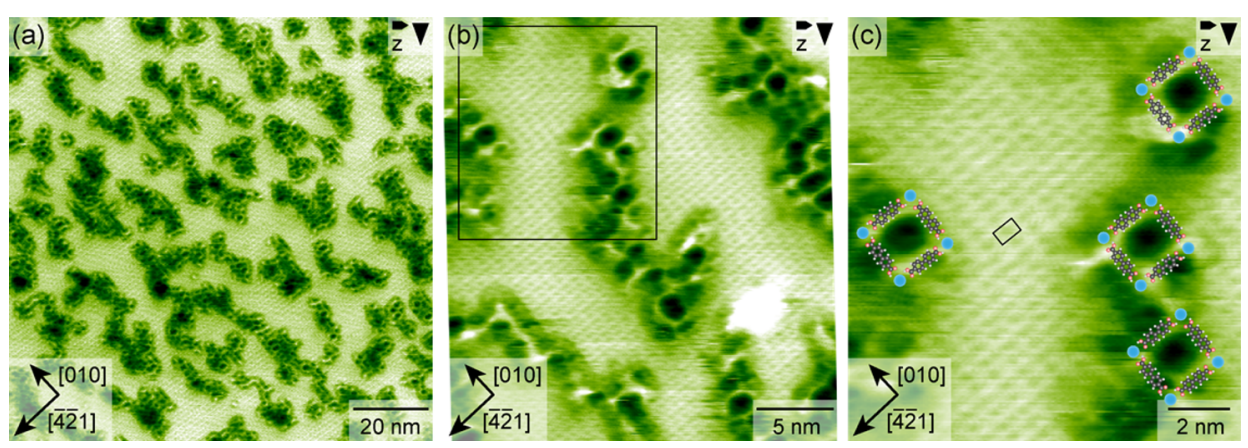

Figure 7. High-resolution NC-AFM images of the first stages of an MOCN formed from BPDCA and Fe on calcite (10.4) revealing simultaneous resolution of the network structure and the underlying calcite lattice (calcite unit cell indicated in (c)). (a) The observed length of the islands is much larger along [010] as compared to the [421] direction. (b) The nodes and linkers of the network are assigned to Fe and BPDCA, respectively, as suggested by the sketch shown in (c), highlighting the positions of some nodes (blue dots representing the metal) and linkers (BPDCA model, not relaxed). For the images shown here, contrast inversion occurred. ${ }^{38,39}$ Therefore, the image contrast is reversed for better comparison with the other FM AFM images shown in this work. 
(a)

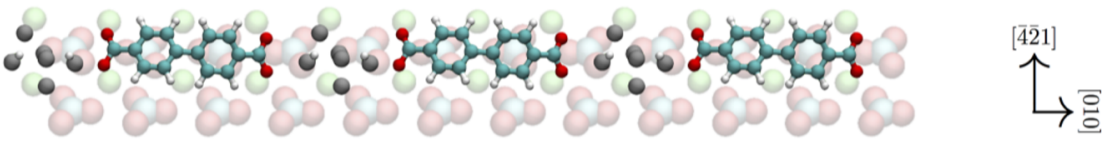

(b)

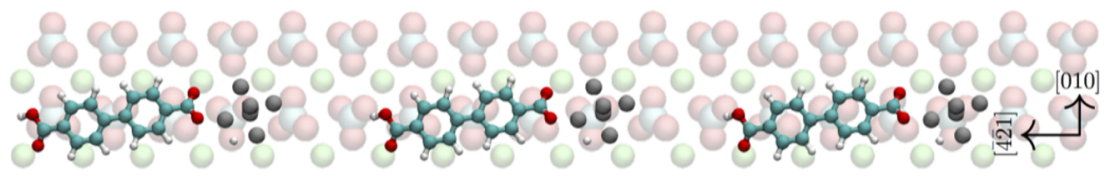

Figure 8. One-dimensional components of the MOCN along (a) the $[010]$ and (b) $[\overline{42} 1]$ surface directions. A chain of BPDCA molecules and Fe ${ }_{6}$ clusters is able to accommodate positions in registry with the surface periodicity along [010]; the same is not true along the [421] direction. In (a) two proton transfer reactions have occurred. Notice that in (b) the whole system has been rotated $90^{\circ}$ counterclockwise for illustration purposes.

an interaction energy between molecule and cluster which is larger than $4 \mathrm{eV}$. This structure can be periodically repeated along the [010] direction conveniently accommodating the underlying calcite periodicity.

The calculations also indicate that a single $\mathrm{Fe}$ atom in the node is not enough to bind the linker molecules even in one direction. This suggests that the nodes contain more than one metal atom, but probably not more than a dozen: the physical size of clusters much larger than this would not be able to accommodate the observed elongation along the [010] surface direction. Along the [ $\overline{42} 1]$ direction, the BPDCA molecule and the metal cluster are not able to form a structure with a matching lattice periodicity (see Figure $8 b$ ). Energetically this complex is more than $2 \mathrm{eV}$ less favorable than the structure along the [010] direction. These simulation results support the hypothesis that the observed preferential extension direction of the MOCN along [010] is due to the templating effect of the substrate.

\section{CONCLUSION}

In conclusion, the first stages of an MOCN on a bulk insulator are created by sequential deposition of BPDCA and Fe on the (10.4) surface of calcite. MOCN structures are obtained only when first adsorbing the molecular linkers and subsequently the metal atoms. The reverse order results in formation of large metal clusters, which appear too inert to dissolve again upon molecule deposition, thus effectively hindering MOCN formation. The resulting MOCN structures are extended only along the [010] surface direction, providing evidence for a substrate templating effect. We speculate that lattice mismatch perpendicular to the [010] direction gives rise to the lateral confinement of the resulting structures, indicating that varying the molecular linker geometry provides strategies toward formation of laterally extended structures.

\section{AUTHOR INFORMATION}

\section{Corresponding Authors}

*E-mail kuehnle@uni-mainz.de, Phone +49 613139 23930; Fax +4961313953930 (experimental part).

*E-mail adam.foster@aalto.fi, Phone +358 50 4333097; Fax +358505389672 (theoretical part).

Notes

The authors declare no competing financial interest.

\section{ACKNOWLEDGMENTS}

We gratefully acknowledge financial support from the EU through grant PAMS (seventh framework program GA 610446), the Academy of Finland through its Centres of
Excellence Program project no. 915804, and the CSC-IT Center for Science Ltd., Espoo, for computational resources.

\section{REFERENCES}

(1) Barth, J. V. Fresh Perspectives for Surface Coordination Chemistry. Surf. Sci. 2009, 603, 1533-1541.

(2) Stepanow, S.; Lin, N.; Barth, J. V. Modular Assembly of LowDimensional Coordination Architectures on Metal Surfaces. J. Phys.: Condens. Matter 2008, 20, 184002.

(3) Umbach, T. R.; Bernien, M.; Hermanns, C. F.; Kruger, A.; Sessi, V.; Fernandez-Torrente, I.; Stoll, P.; Pascual, J. I.; Franke, K. J.; Kuch, W. Ferromagnetic Coupling of Mononuclear Fe Centers in a SelfAssembled Metal-Organic Network on Au(111). Phys. Rev. Lett. 2012, $109,5$.

(4) Seitsonen, A. P.; Lingenfelder, M.; Spillmann, H.; Dmitriev, A.; Stepanow, S.; Lin, N.; Kern, K.; Barth, J. V. Density Functional Theory Analysis of Carboxylate-Bridged Diiron Units in Two-Dimensional Metal-Organic Grids. J. Am. Chem. Soc. 2006, 128, 5634-5635.

(5) Fabris, S.; Stepanow, S.; Lin, N.; Gambardella, P.; Dmitriev, A.; Honolka, J.; Baroni, S.; Kern, K. Oxygen Dissociation by Concerted Action of Di-Iron Centers in Metal-Organic Coordination Networks at Surfaces: Modeling Non-Heme Iron Enzymes. Nano Lett. 2011, 11, 5414-5420.

(6) Furukawa, S.; Reboul, J.; Diring, S.; Sumida, K.; Kitagawa, S. Structuring of Metal-Organic Frameworks at the Mesoscopic/ Macroscopic Scale. Chem. Soc. Rev. 2014, 43, 5700-5734.

(7) Yaghi, O. M.; O’Keeffe, M.; Ockwig, N. W.; Chae, H. K.; Eddaoudi, M.; Kim, J. Reticular Synthesis and the Design of New Materials. Nature 2003, 423, 705-714.

(8) Furukawa, H.; Cordova, K. E.; O’Keeffe, M.; Yaghi, O. M. The Chemistry and Applications of Metal-Organic Frameworks. Science 2013, 341, 1230444.

(9) Furukawa, H.; Mueller, U.; Yaghi, O. M. "Heterogeneity within Order" in Metal-Organic Frameworks. Angew. Chem., Int. Ed. 2015, 54, 3417-3430.

(10) Wang, Z. F.; Liu, Z.; Liu, F. Organic Topological Insulators in Organometallic Lattices. Nat. Commun. 2013, 4, 1471.

(11) Lin, N.; Stepanow, S.; Vidal, F.; Barth, J. V.; Kern, K. Manipulating 2d Metal-Organic Networks Via Ligand Control. Chem. Commun. 2005, 1681-1683.

(12) Schlickum, U.; Decker, R.; Klappenberger, F.; Zoppellaro, G.; Klyatskaya, S.; Ruben, M.; Silanes, I.; Arnau, A.; Kern, K.; Brune, H.; Barth, J. V. Metal-Organic Honeycomb Nanomeshes with Tunable Cavity Size. Nano Lett. 2007, 7, 3813-7.

(13) Stepanow, S.; Lingenfelder, M.; Dmitriev, A.; Spillmann, H.; Delvigne, E.; Lin, N.; Deng, X.; Cai, C.; Barth, J. V.; Kern, K. Steering Molecular Organization and Host-Guest Interactions Using TwoDimensional Nanoporous Coordination Systems. Nat. Mater. 2004, 3, 229-233.

(14) Lin, T.; Wu, Q.; Liu, J.; Shi, Z. L.; Liu, P. N.; Lin, N. Thermodynamic Versus Kinetic Control in Self-Assembly of Zero-, One-, Quasi-Two-, and Two-Dimensional Metal-Organic Coordination Structures. J. Chem. Phys. 2015, 142, 101909. 
(15) Lingenfelder, M. A.; Spillmann, H.; Dmitriev, A.; Stepanow, S.; Lin, N.; Barth, J. V.; Kern, K. Towards Surface-Supported Supramolecular Architectures: Tailored Coordination Assembly of 1,4Benzenedicarboxylate and $\mathrm{Fe}$ on $\mathrm{Cu}(100)$. Chem. - Eur. J. 2004, 10, 1913-1919.

(16) Stepanow, S.; Lin, N.; Barth, J. V.; Kern, K. Surface-Template Assembly of Two-Dimensional Metal-Organic Coordination Networks. J. Phys. Chem. B 2006, 110, 23472-23477.

(17) Dmitriev, A.; Spillmann, H.; Lin, N.; Barth, J. V.; Kern, K. Modular Assembly of Two-Dimensional Metal-Organic Coordination Networks at a Metal Surface. Angew. Chem., Int. Ed. 2003, 42, 26702673.

(18) Pivetta, M.; Pacchioni, G. E.; Fernandes, E.; Brune, H. Temperature-Dependent Self-Assembly of Nc-Ph-5-Cn Molecules on $\mathrm{Cu}(111)$. J. Chem. Phys. 2015, 142, 101928.

(19) Rodriguez-Fernandez, J.; Lauwaet, K.; Herranz, M. A.; Martin, N.; Gallego, J. M.; Miranda, R; Otero, R. Temperature-Controlled Metal/Ligand Stoichiometric Ratio in Ag-Tcne Coordination Networks. J. Chem. Phys. 2015, 142, 101930.

(20) Studener, F.; Muller, K.; Marets, N.; Bulach, V.; Hosseini, M. W.; Stohr, M. From Hydrogen Bonding to Metal Coordination and Back: Porphyrin-Based Networks on $\mathrm{Ag}(111)$. J. Chem. Phys. 2015, 142, 101926.

(21) Urgel, J. I.; Schwarz, M.; Garnica, M.; Stassen, D.; Bonifazi, D.; Ecija, D.; Barth, J. V.; Auwaerter, W. Controlling Coordination Reactions and Assembly on a $\mathrm{Cu}(111)$ Supported Boron Nitride Monolayer. J. Am. Chem. Soc. 2015, 137, 2420-2423.

(22) Shekhah, O.; Liu, J.; Fischer, R. A.; Wöll, C. Mof Thin Films: Existing and Future Applications. Chem. Soc. Rev. 2011, 40, 10811106.

(23) Jain, R.; Kabir, K.; Gilroy, J. B.; Mitchell, K. A. R.; Wong, K.-c.; Hicks, R. G. High-Temperature Metal-Organic Magnets. Nature 2007, 445, 291-294.

(24) Barth, C.; Henry, C. R. High-Resolution Imaging of Gold Clusters on $\mathrm{Kbr}(001)$ Surfaces Investigated by Dynamic Scanning Force Microscopy. Nanotechnology 2004, 15, 1264-1272.

(25) Barth, C.; Henry, C. R. Gold Nanoclusters on Alkali Halide Surfaces: Charging and Tunneling. Appl. Phys. Lett. 2006, 89, 252119.

(26) Bauer, E. Phänomenologische Theorie Der Kristallabscheidung an Oberflächen. I. Z. Kristallogr. 1958, 110, 372-394.

(27) Abel, M.; Clair, S.; Ourdjini, O.; Mossoyan, M.; Porte, L. Single Layer of Polymeric Fe-Phthalocyanine: An Organometallic Sheet on Metal and Thin Insulating Film. J. Am. Chem. Soc. 2011, 133, 12031205.

(28) Kittelmann, M.; Rahe, P.; Kühnle, A. Molecular Self-Assembly on an Insulating Surface: Interplay between Substrate Templating and Intermolecular Interactions. J. Phys.: Condens. Matter 2012, 24, 354007-354013.

(29) Perdew, J. P.; Burke, K.; Ernzerhof, M. Generalized Gradient Approximation Made Simple. Phys. Rev. Lett. 1996, 77, 3865-3868.

(30) Grimme, S.; Antony, J.; Ehrlich, S.; Krieg, H. A Consistent and Accurate $\mathrm{Ab}$ Initio Parametrization of Density Functional Dispersion Correction (Dft-D) for the 94 Elements H-Pu. J. Chem. Phys. 2010, 132, 154104.

(31) Kresse, G.; Furthmüller, J. Efficient Iterative Schemes for Ab Initio Total-Energy Calculations Using a Plane-Wave Basis Set. Phys. Rev. B: Condens. Matter Mater. Phys. 1996, 54, 11169-11186.

(32) Blöchl, P. E. Projector Augmented-Wave Method. Phys. Rev. B: Condens. Matter Mater. Phys. 1994, 50, 17953-17979.

(33) Monkhorst, H. J.; Pack, J. D. Special Points for Brillouin-Zone Integrations. Phys. Rev. B 1976, 13, 5188-5192.

(34) VandeVondele, J.; Krack, M.; Mohamed, F.; Parrinello, M.; Chassaing, T.; Hutter, J. Quickstep: Fast and Accurate Density Functional Calculations Using a Mixed Gaussian and Plane Waves Approach. Comput. Phys. Commun. 2005, 167, 103-128.

(35) Lippert, G.; Hutter, J.; Parrinello, M. A Hybrid Gaussian and Plane Wave Density Functional Scheme. Mol. Phys. 1997, 92, 477487.
(36) Goedecker, S.; Teter, M.; Hutter, J. Separable Dual-Space Gaussian Pseudopotentials. Phys. Rev. B: Condens. Matter Mater. Phys. 1996, 54, 1703-1710.

(37) Henkelman, G.; Uberuaga, B. P.; Jonsson, H. A Climbing Image Nudged Elastic Band Method for Finding Saddle Points and Minimum Energy Paths. J. Chem. Phys. 2000, 113, 9901-9904.

(38) Rahe, P.; Bechstein, R.; Schütte, J.; Ostendorf, F.; Kühnle, A. Repulsive Interaction and Contrast Inversion in Noncontact Atomic Force Microscopy Imaging of Adsorbates. Phys. Rev. B: Condens. Matter Mater. Phys. 2008, 77, 195410.

(39) Loske, F.; Rahe, P.; Kühnle, A. Contrast Inversion in NonContact Atomic Force Microscopy Imaging of $\mathrm{C}_{60}$ Molecules. Nanotechnology 2009, 20, 264010. 